\title{
Considerações adaptativas e morfológicas dos ossos do membro torácico do Tapirus terrestris (Perissodactyla, tapiridae)
}

\author{
Saulo Gonçalves Pereira ${ }^{*}$, Daniela Cristina Silva Borges ${ }^{2}$, \\ Felipe César de Araújo Machado ${ }^{3}$, Alex Rodrigo Borges ${ }^{1,2}$, \\ André Luiz Quagliatto Santos ${ }^{4}$
}

\begin{abstract}
RESUMO
Tapirus terrestris é um dos maiores mamíferos das Américas, distribuindose desde o Sul do Brasil e da Argentina até o norte do continente sulamericano. Objetivou-se, com este estudo, traçar um breve histórico do surgimento dos tapirídeos através de uma revisão de literatura e apresentar as adaptações morfológicas e ambientais dos ossos do cíngulo escapular, do braço, do antebraço e da mão do T. terrestris. Utilizaram-se quatro esqueletos de antas doados ao Laboratório de Ensino e Pesquisa em Animais Silvestres da Universidade Federal de Uberlândia. Os ossos foram minuciosamente analisados e descritos. Os tapirídeos surgiram na Terra no início do Pleistoceno e na América do Sul no Mioceno. Os ossos do membro torácico são: escapula, úmero, rádio, ulna, ossos do carpo, metacarpo e falanges. Os achados permitiram verificar que esses ossos são protuberantes e resistentes, com acidentes que, aparentemente, auxiliam no deslocamento cursorial. Por ser um animal cursorial, o T. terrestris tem adaptações morfológicas específicas que se relacionam ao seu nicho e habitat.
\end{abstract}

Palavras-chave: Cursoriais, Mamíferos, Morfologia descritiva, Evolução, Tapirus terrestris.

$$
* * *
$$

\section{Introdução}

Os tapirídeos são classificados na ordem Perissodactyla, subordem Ceratomorpha, superfamília Tapiroides, família Tapiridae e gênero Tapirus (PADILLA et al., 1994). Esses mamíferos têm um número ímpar de dedos, um ou

1 Universidade Federal de Uberlândia e Faculdade Patos de Minas

${ }^{2}$ Universidade Federal de Uberlândia e Faculdade Cidade de João Pinheiro

${ }^{3}$ Unipam

${ }^{4}$ Universidade Federal de Uberlândia - Famev/Lapas

* Corresponding author: Saulo Gonçalves Pereira; endereço: Laboratório de Ensino e Pesquisa em Animais Silvestres (Lapas/(UFU), Rua Piauí 1302, Umuarama, Uberlândia-MG, 38400-902, Brasil; telefone: 34 32258404; e-mail: saulobiologo@yahoo.com.br 
três, cada um com um casco (HICKMAN, ROBERTS, LARSON, 2009). Já as antas têm quatro dedos na mão (STORER et al., 2000).

As antas tiveram sua origem, segundo Radinsky (1963), na E,uropa no período Pleistoceno. Na América do Sul o seu registro ocorre a partir do Mioceno (HOLANDA, 2007). O T. terrestris é um dos maiores mamíferos das Américas, distribuindo-se desde o Sul do Brasil e da Argentina até o norte do continente sulamericano. No Brasil, as antas existem em quase todos os biomas, exceto nos Pampas (PADILLA et al., 1994). São animais herbívoros, encontrados acompanhados apenas durante a época de acasalamento e na amamentação (MEDICI, 2011).

A anatomia, de acordo com Hildebrand e Goslow (2006), ocupa-se do estudo das estruturas do corpo, todavia, conceitualmente, o termo morfologia já é mais abrangente. Esta procura estudar os "fatos" anatômicos, ou seja, tenta explicar os padrões estruturais à luz da evolução, da paleontologia, da taxonomia, dentre outras ciências.

A evolução é o processo pelo qual ocorrem mudanças ou transformações nos seres vivos ao longo do tempo, dando origem a espécies novas. Segundo Hickman, Roberts, Larson (2009), o registro fóssil permite perceber as evoluções através do tempo. As espécies surgem e desaparecem na escala de tempo, e, nesse sentido, a seleção natural fornece explicações para as adaptações surgidas nas espécies, inclusive em sua anatomia.

A variabilidade, o aparecimento e o aumento dos seres devem-se à ocorrência de mutações, à seleção natural e à reprodução sexuada. Acredita-se que a problemática da especiação seja a máxima central na teoria evolutiva (VANZOLINI, 1992).

Nessa perspectiva, a anatomia comparada, segundo Ridley (2006), ocupa-se de comparar a estrutura anatômica de diferentes grupos de animais para assim estabelecer relações filogenéticas e colaborar para estudos morfológicos.

Parte-se da hipótese de que o meio ambiente tenha grande relação no processo evolutivo, trazendo, em uma escala temporal, adaptações para as espécies, ou mesmo a extinção destas em função das diversas pressões antrópicas que sofrem. No caso do T. terrestres, acredita-se que ele tenha se adaptado ao seu habitat. Notadamente, a morfologia contribui de forma memorável com dados das 
espécies, sobretudo no que tange ao conhecimento da anatomia e a suas aplicações veterinárias, embriológicas, ecológicas, dentre outras, pois o conhecimento sobre a morfologia adaptativa dos animais pode fornecer evidências nos processos de evolução dos organismos e em sua adaptação ao meio.

Sendo assim, objetivou-se neste artigo traçar um breve histórico do surgimento dos tapirídeos ao longo da escala geológica do tempo através de uma revisão de literatura e apresentar as adaptações morfológicas e ambientais dos ossos do cíngulo escapular, do antebraço e da mão do T. terrestris.

\section{Material e métodos}

O trabalho está divido em duas partes: a primeira apresenta um levantamento acerca da história evolutiva dos tapirídeos, e a segunda faz uma análise das estruturas ósseas dos ossos do cíngulo escapular, do braço e da mão do T. terrestris com o objetivo de fazer uma relação com o habitat.

Inicialmente, foi feito um levantamento bibliográfico de forma qualitativa e explanatória, que, segundo Minayo (1994), é um estudo que se ocupa do levantamento e da releitura de escritos sem preocupação com análises estatísticas. Para tanto, foi realizada uma ampla pesquisa bibliográfica. Os textos e escritos que abordam a origem do T. terrestris foram fichados.

Para as análises foram utilizadas quatro peças anatômicas do esqueleto do T. terrestris. Esses espécimes foram doados por um criadouro, após óbito natural sem trauma, ao Laboratório de Ensino e Pesquisa em Animais Silvestres da Universidade Federal de Uberlândia (Lapas-UFU). As peças são de animais adultos. A pesquisa foi autorizada pelo Conselho de Ética para a Utilização de Animais da UFU (CEUA), sob o parecer 069/12, e está de acordo com a Instrução Normativa 154/2007 do Ibama.

Os ossos foram identificados, minuciosamente analisados, descritos e comparados com a literatura pertinente. A nomenclatura adotada está de acordo com o International Committee on Veterinary Gross Anatomical 
Nomenclature (2012). As imagens foram registradas com câmera digital (Kodak Easy Share C182, 12 mega pixel) e tratadas pelo software Adobe Photoshop CC 14.

\section{Resultados e discussão}

\subsection{A evolução dos vertebrados e dos tapirídeos}

Os vertebrados abrangem cerca de 50.000 espécies descritas atualmente, com representantes aquáticos e terrestres. Os vertebrados são triblásticos, celomados e deuterostômicos. Nos indivíduos adultos, a notocorda é substituída pela coluna vertebral. As evidências fósseis indicam que os vertebrados evoluíram do ambiente marinho durante o Cambriano, precisamente no Paleozoico Inferior (STORER et al., 2000).

A deriva continental é uma teoria criada por Alfred Wegener, em que se apresenta que há aproximadamente 200 milhões de anos não existia separação entre os continentes, havendo apenas uma única massa continental denominada Pangeia e um oceano único denominado Pantalassa (MOLEDO et al., 2010). Após dois milhões de anos houve uma fragmentação, dando origem a dois megacontinentes, Laurásia e Godwana, e a partir daí os continentes foram se distanciando e se amoldando às conformações atuais. Segundo Salgado-Labouriau (1994), a movimentação dos continentes ao longo do tempo trouxe efeitos importantes nos processos evolutivos dos vertebrados. O mais patente é a ralação entre a localização das massas de Terra e seus climas, bem como as adaptações das estruturas dos seus corpos em função do desenvolvimento dos seus nichos.

Segundo Pough, Janis e Heiser (2008), houve profundas mudanças na estrutura da crosta terrestre durante os 500 mil anos de história dos vertebrados. Dessa maneira, a deriva continental teve grande influência nos processos evolutivos e adaptativos dos vertebrados, com a fixação de suas características devido ao isolamento em uma escala mundial. 
Dentro do filo dos cordados, subfilo dos vertebrados, existem os tetrápodes, que têm, ainda hoje, sua origem discutida. O fóssil mais antigo, segundo Pough, Heiser e Mcfarland (1993), é conhecido como Ichthyostega e datado do Devoniano Médio e Superior (aproximadamente 350 milhões de anos atrás), com ocorrência na Groenlândia e Austrália. Os últimos estudos para o surgimento dos tetrápodes têm apontado que eles surgiram a partir dos peixes Osteolepiformes, devido à semelhança na forma do crânio e dos ossos dos membros (POUGH, JANIS, HEISER, 2008).

Já os estudos de Redford (1989) apontam que, devido a frequentes buscas e coletas de fósseis, existe atualmente uma quantidade considerável de depósitos de fósseis do Devoniano Médio ao Superior (360-375 milhões de anos atrás), contendo fósseis de tetrápodes como também de peixes, que seriam mais proximamente relacionados aos primeiros tetrápodes. Ainda segundo os mesmos autores, as espécies terrestres são encontradas em depósitos do Carbonífero (cerca de 330 milhões de anos atrás).

Acredita-se que no período Devoniano o meio aquático estava abarrotado de diferentes espécies de peixes, assim a competição e a predação podem ter conduzido esses peixes para um novo ambiente, com novos recursos a serem explorados. Existem muitas divergências com relação à origem dos mamíferos, todavia não se pode acreditar que ela tenha ocorrido como um acontecimento súbito e instantâneo no final do Triásico. Acreditase que eles tenham tido origem a partir dos répteis sinapsídeos e que as suas estruturas anatômicas evoluíram em conjunto durante todo o período que vai do Permiano ao Triásico (HICKMAN, ROBERTS, LARSON, 2009).

Ainda segundo esse autor, acredita-se que os mamíferos tiveram origem em um grupo já extinto de répteis sinapsídeos, os denominados terapsídeos, que tiverem origem entre o Permiano Médio e o final do Triásico. Os atuais tetrápodes originaram-se em mamíferos reptilianos. Pela análise de seus fósseis, estes tinham esqueleto e dentição parecidos com os 
dos mamíferos e desenvolveram o controle endotérmico da temperatura corporal (HICKMAN, ROBERTS, LARSON, 2009).

Com relação à origem da família Tapiridae na Terra, as evidências demonstram que ocorreu a partir do início do Pleistoceno. O seu registro é datado possivelmente da época do Eoceno na América do Norte (HUBBE, 2008). Já na América do Sul, a origem é possivelmente o final da época do Mioceno (CAMPBELL et al., 1936). As evidências e os achados são em função de estudos paleontológicos.

De acordo com os estudos de Hubbe (2008), acredita-se que essa irradiação intercontinental da família Tapiridae seja em função da interconexão continental, o que reforça a teoria da Deriva Continental.

Segundo Hubbe (2008), existem ainda discussões sobre as relações filogenéticas de vida no Pleistoceno, sobretudo no que tange à irradiação e à origem das antas. Acredita-se que elas tenham chegado à América do Sul durante o Pleistoceno Inferior, bem como na América Central e na Ásia (EISENBERG et al., 1999).

\subsection{Ossos do Tapirus terrestris}

Os ossos do cíngulo escapular, do braço e do antebraço do T. terrestris são a escápula, o úmero, o rádio e a ulna; os da mão são os ossos do carpo, os metacarpos, as falanges e os sesamóides. As fotos a seguir apresentam os ossos, bem como seus acidentes.

A escápula do T. terrestris é um osso plano, com aspecto ovalado e irregular em relação às suas extremidades proximal e distal, encontrando-se preso obliquamente à parte cranial da parede torácica e articulando-se distalmente com a cabeça do úmero através da cavidade glenóide, funcionando como um conector. No equino (Equus caballus), a escápula é um osso triangular. Em T. terrestris apresenta duas faces (medial e lateral), duas margens (cranial e caudal) e dois ângulos (cranial, caudal). Segundo 
Holbrook (2012) e Pereira et al. (2015), existe uma proximidade filogenética entre essas espécies corroborando semelhanças anatômicas.

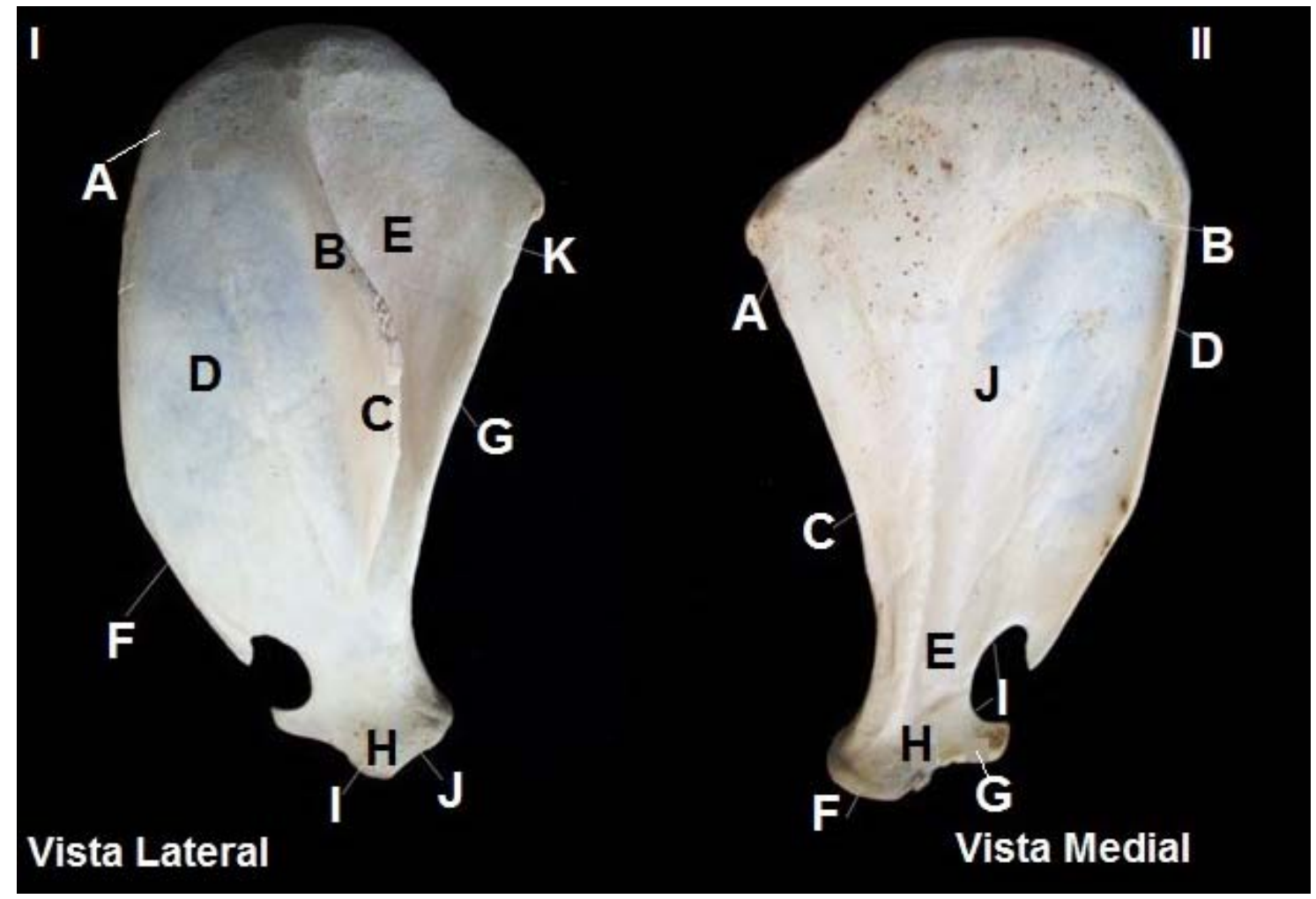

Figura 1 - Fotografia da escápula, I vista lateral; II vista medial. (Fonte: Acervo particular. Uberlândia, 2012).

Legenda: I: Vista lateral - (A) ângulo cranial; (B) espinha da escápula; (C) tuberosidade da escápula; (D) fossa supraespinhal; (E) fossa infraespinhal; (F) margem cranial; (G) margem caudal; (H) forame nutrício; (I) tuberosidade supraglenoidal; (J) cavidade glenóide; (K) ângulo caudal.

II: Vista Medial - (A) ângulo caudal; (B) ângulo cranial; (C) margem caudal; (D) margem cranial; (E) colo da escápula; (F) cavidade glenóide; (G) tuberosidade supraglenoidal; (H) processo coracóide; (I) incisura na margem lateral distal; (J) fossa subescapular. 


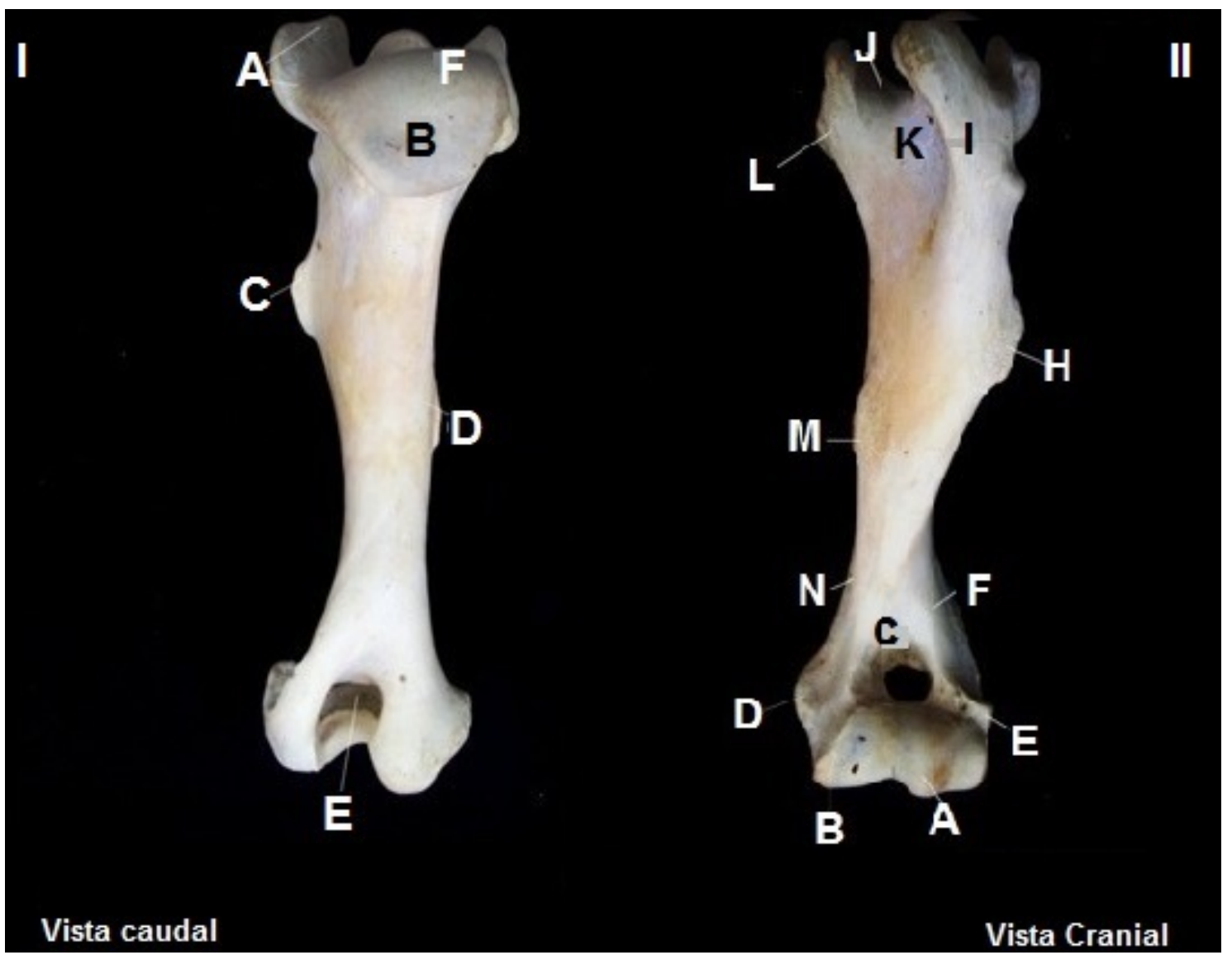

Figura 2 - Fotografia do úmero do T. terrestris. (Fonte: Acervo particular. Uberlândia, 2012).

Legenda: I: Vista Caudal - (A) tuberosidade maior; (B) colo; (C) tuberosidade deltóidea; (D) tuberosidade para o músculo redondo menor (E) fossa do olecrano (F) cabeça do úmero.

II: Vista Cranial - (A) capítulo; (B) tróclea; (C) fossa radial; (D) epidicôndilo medial; (E) epicôndilo lateral; (F) crista do epicôndilo lateral; (G) sulco para o músculo braquial; (H) tuberosidade deltóidea; (I) tuberosidade maior; (J) sulco intertuberal; (K) forame nutrício proximal; (L) tuberosidade menor; (M) tuberosidade para o músculo redondo maior; (N) forame nutrício distal.

A ulna de T. terrestris articula-se proximalmente com o úmero e se encaixa na fossa do olécrano. É constituída das extremidades proximal e distal (epífises) e do corpo (diáfise). É um osso longo e largo, porém menos volumoso se comparado ao rádio. A ulna do T. terrestris, assim como a dos equinos, é maior se comparada a dos ruminantes.

O rádio é um osso longo e situa-se cranialmente à ulna. No T. terrestris é constituído de cabeça, colo, corpo e extremidade articular cárpica ou tróclea. Diferentemente dos equinos, não é curvo e articula-se proximalmente com o úmero e distalmente com o carpo. 


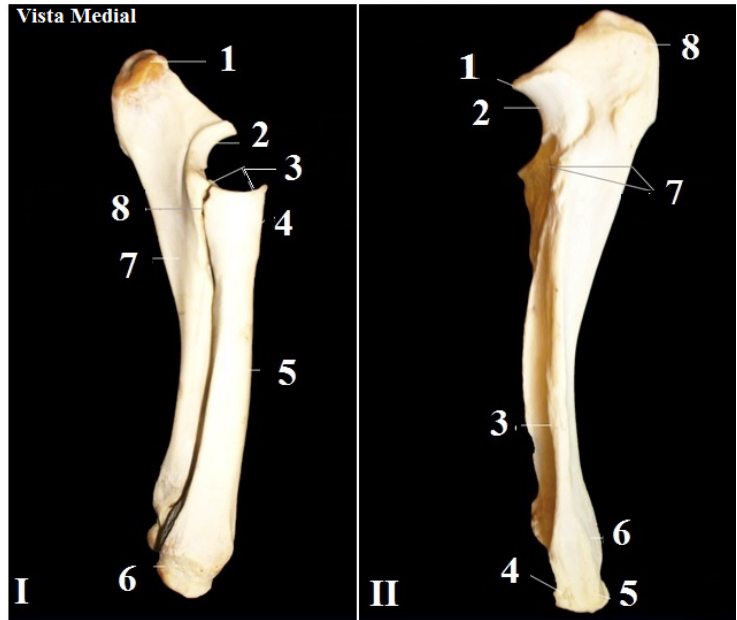

Figura 3 - Fotografia dos ossos rádio e ulna do T. terrestris, vista medial. (Fonte: Acervo particular. Uberlândia, 2012).

Legenda: I: Ulna e rádio - (1) tuberosidade do olécrano; (2) incisura troclear; (3) fóvea da cabeça do rádio; (4) tuberosidade do rádio; (5) corpo do rádio; (6) superfície articular cárpica; (7) corpo da ulna; (8) espaço interósseo. II: Ulna - (1) processo ancôneo; (2) incisura troclear; (3) crista da ulna; (4) forames; (5) crista transversa; (6) extremidade distal da ulna; (7) espaço interósseo; (8) tuberosidade do olécrano.

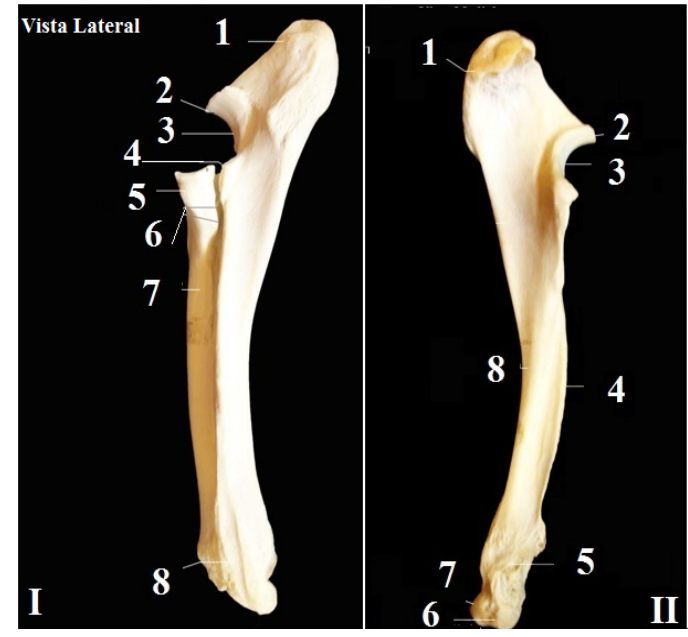

Figura 4 - Fotografia dos ossos rádio e ulna do T. terrestris, vista lateral. (Fonte: Acervo particular. Uberlândia, 2012).

Legenda: I: Rádio e ulna - (1) tuberosidade do olécrano; (2) processo ancôneo; (3) fissura troclear; (4) espaço interósseo; (5) tuberosidade do rádio; (6) espaço interósseo; (7) corpo do rádio; (8) sulco do tendão extensor digital comum. II: Ulna - (1) tuberosidade do olécrano; (2) processo ancôneo; (3) incisura troclear; (4) crista do corpo da ulna; (5) extremidade distal da ulna; (6) superfície articular cárpica; (7) crista transversa; (8) sulco do tendão extensor digital comum.

Os ossos do carpo no T. terrestris são um conjunto de sete ossos curtos divididos em duas fileiras, proximal e distal, assim como nos bovinos e equinos, e cada osso do carpo apresenta-se de maneira diferente em cada espécie de animal. 


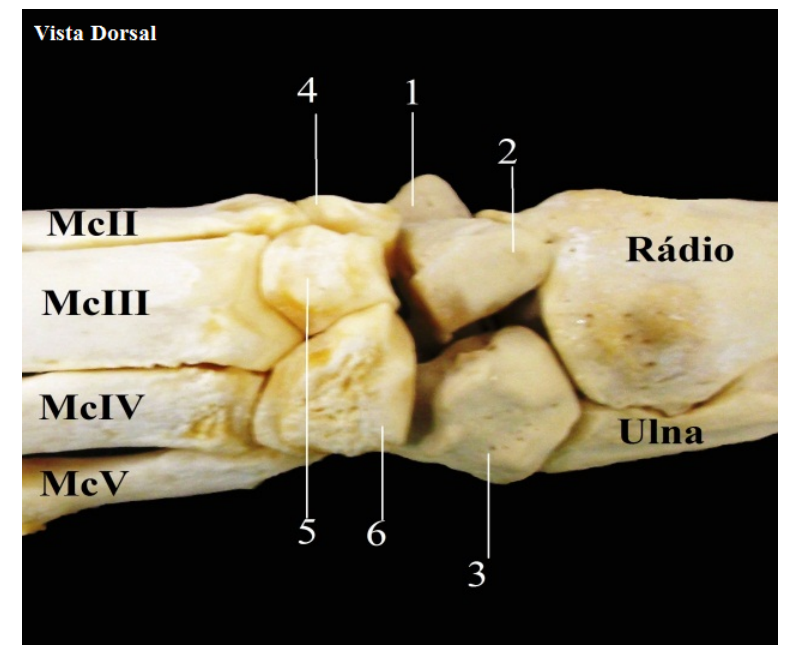

Figura 5 - Fotografia dos ossos do carpo e metacarpo do T. terrestris, vista dorsal. (Fonte: Acervo particular. Uberlândia, 2013).

Legenda: (1) carpo radial; (2) carpo intermédio; (3) carpo ulnar; (4) cárpico II; (5) cárpico III; (6) cárpico IV; (McII) metacarpo II; (McIII) metacarpo III; (McIV) metacarpo IV; (McV) metacarpo V.

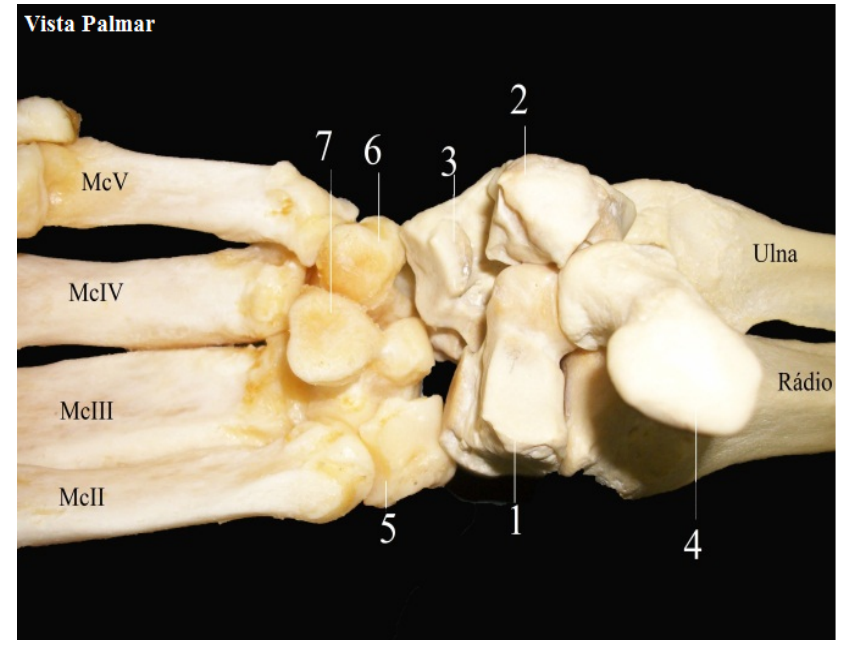

Figura 6 - Fotografia dos ossos do carpo e do metacarpo do T. terrestris, vista palmar. (Fonte: Acervo particular. Uberlândia, 2013).

Legenda: (1) carpo radial; (2) carpo intermédio; (3) carpo ulnar; (4) carpo acessório; (5) cárpico II; (6) cárpico IV; (7) cárpico III; (McII) metacarpo II; (McIII) metacarpo III; (McIV) metacarpo IV; (McV) metacarpo V.

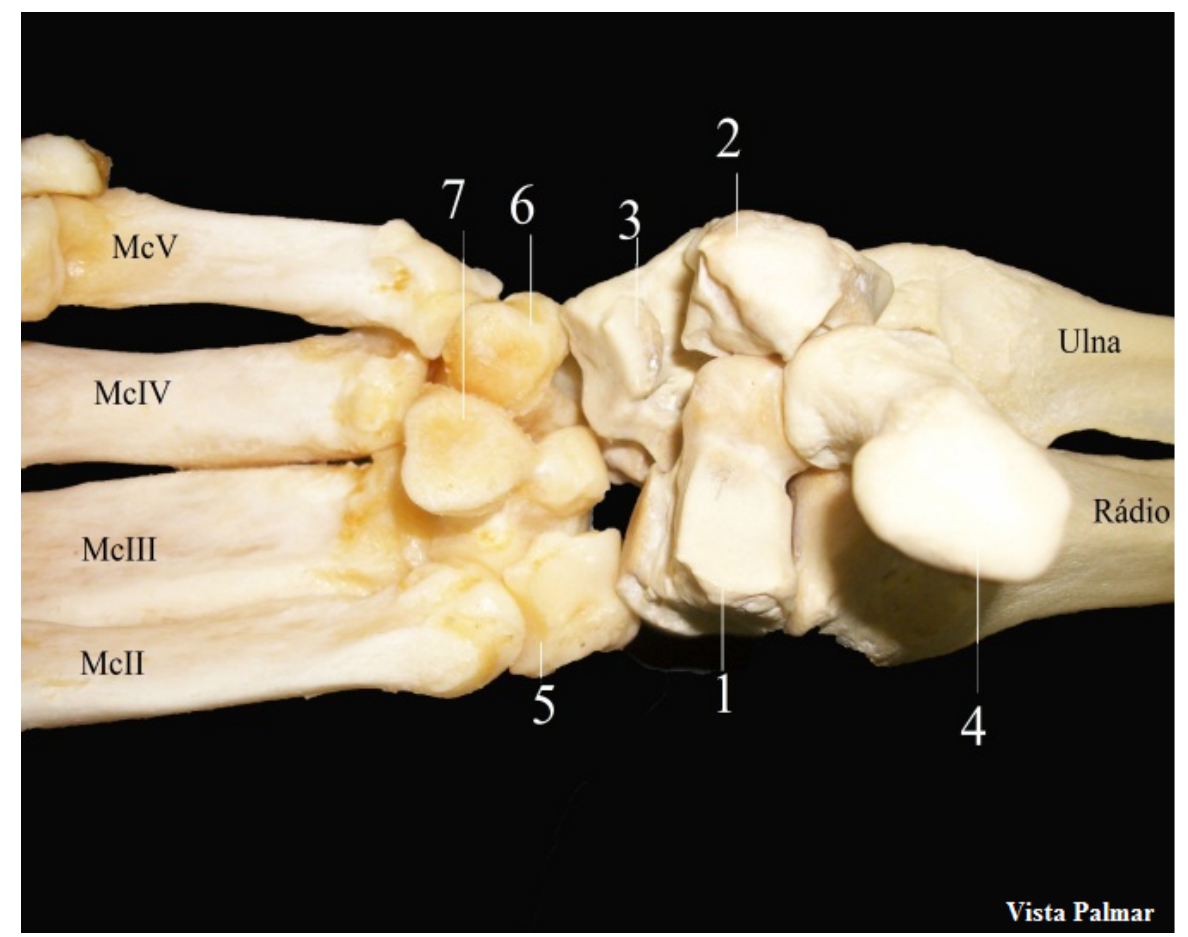

Figura 4 - Fotografia dos ossos do carpo e do metacarpo do T. terrestris, vista palmar. (Fonte: Acervo particular. Uberlândia, 2013). Legenda: (1) carpo radial; (2) carpo intermédio; (3) carpo ulnar; (4) cárpico acessório; (5) cárpico II; (6) cárpico IV; (7) cárpico III; (McII) metacarpo II; (McIII) metacarpo III; (McIV) metacarpo IV; (McV) metacarpo V. 


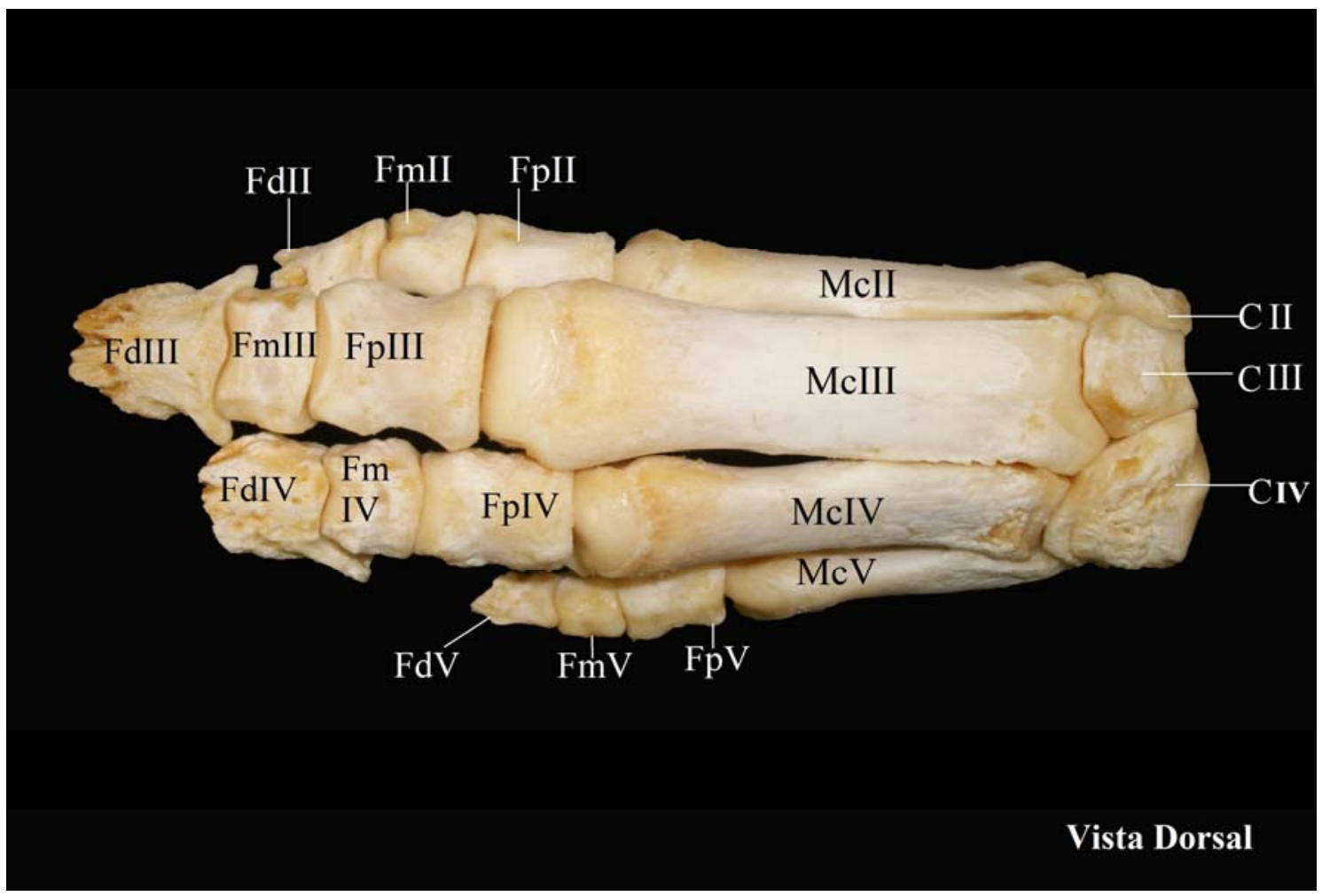

Figura 5 - Fotografia dos ossos da mão do T. terrestris, vista dorsal. (Fonte: Acervo particular. Uberlândia, 2013). Legenda: (CII) cárpico II; (CIII) cárpico III; (CIV) cárpico IV; (McII) metacarpo II; (McIII) metacarpo III; (McIV) metacárpico IV; (McV) metacarpo V; (1) e (2) sesamóides dedo V; (3) e (4) sesamóides dedo IV; (5) e (6) sesamóides dedo III; (7) e (8) sesamóides dedo II; (FpV) falange proximal do dedo $\mathrm{V} ;(\mathrm{FmV})$ falange média do dedo $\mathrm{V} ;(\mathrm{FdV})$ falange distal do dedo $\mathrm{V}$; $(\mathrm{FpIV})$ falange proximal do dedo IV; (FmIV) falange média do dedo IV; (FdIV) falange distal do dedo IV; (FpIII) falange proximal do dedo III; (FmIII) falange média do dedo III; (FdIII) falange distal do dedo III; (FpII) falange proximal do dedo II; (FmII) falange média do dedo II; (FdII) falange distal do dedo II.

Os ossos do metacarpo do T. terrestris estão dispostos em quatro (Figuras 4, 5 e 6), assim denominados metacarpo (Mc) - McII, McIII, McIV e $\mathrm{McV}$-, o que difere esse tipo de Perissodactyla dos demais, que apresentam uma quantidade ímpar de dedos. Todavia, filogeneticamente, estão incluídos nessa ordem (CAMPBELL, 1936). Os ossos do metacarpo têm uma superfície articular lisa, são longos, com o corpo achatado e articulam-se com as falanges proximais.

O T. terrestris é classificado, com relação ao seu deslocamento, como cursorial, ou seja, animais que têm a capacidade de longo deslocamento por terra, com passadas longas. Frequentemente são animais de médio a grande porte (HILDEBRAND e GOSLOW, 2006). 
No caso do T. terrestris, esses animais são classificados como digitígrados cursoriais, por andarem apoiados sobre os dedos. Acredita-se que os ossos dos dedos da mão do T. terrestris (Figuras 4, 5 e 6) sejam adaptados para essa postura, pois apresentam acidentes ósseos específicos e com características bastante proeminentes.

A grande vantagem adaptativa dos animais cursoriais é poder desenvolver seu nicho por longas áreas, inclusive migrando à procura de áreas associadas a cursos d'água. Outra habilidade dos cursores é a resistência (HILDEBRAND e GOSLOW, 2006). Percebe-se que os ossos do T. terrestres são alongados, proeminentes e resistentes, além de terem epífises desenvolvidas e fortes.

O T. terrestris tem seu habitat associado às áreas próximas a cursos d'água, áreas descampadas e formações florestais (FINNEGAN et al., 1993; MEDICI, 2001). São animais herbívoros (ZORZI, 2009) e esse hábito exerce uma grande influência nas comunidades florestais, alterando sua estrutura, dinâmica e diversidade biológica, pois as antas atuam como dispersores e predadores de variadas espécies vegetais (NARANJO e CRUZ, 1998). As interações morfológicas dos cursoriais com o meio ambiente reforçam que possa haver uma manutenção mutualística para manter os padrões e os processos ecológicos entre dispersores e sementes, sobretudo da megaflora, devido ao hábito de deslocamento por grandes áreas realizado pelo $T$. terrestris (FRAGOSO e HUFFMAN, 2000).

Os Perissodactyla têm a capacidade de correr e trotar (POUGH; JANIS; HEISER, 2008). Ao superar a inércia, esse animal tem que impulsionar o corpo sustentado em seus dedos, ou seja, a postura digitígrada. Como são animais ungulados, a postura é denominada "ugulígrida" (HILDEBRAND, 1995). Percebe-se que os ossos do braço do $T$. terrestris são alongados e fortes, demonstrando, assim, adaptações a esse movimento cursorial (HERNANDEZ-DIVERS, 2007). 
Segundo Hildebrand e Goslow (2006), quanto mais longa a pata, mais longo o passo. As patas dianteiras do T. terrestris são alongadas, elas têm quatro dedos, o que auxilia, nesse sentido, em seu deslocamento (CAMPBELL, 1936). Outra característica é que o rádio e o úmero são proporcionais, tanto em relação às suas dimensões longitudinais como no tocante aos diâmetros, reforçando a ideia de que os cursoriais são adaptados ao passo. Esses ossos são sulcados em suas epífises (HILDEBRAND e GOSLOW, 2006). Outra evidência são os ossos metacarpais, especificamente o dedo III, que também é mais longo (GAMBARYAN, 1974). O cíngulo escapular não possui o osso da clavícula, o que auxilia no deslocamento por longas distâncias (HILDEBRAND e GOSLOW, 2006).

Com relação à quantidade de dedos, a anta tem quatro dedos na mão, que, como mencionado, auxiliam no deslocamento e na postura de descanso. Já os carnívoros, ao longo da evolução, geralmente perdem a funcionalidade do dedo um, no entanto comprimem os dedos em conjunto em vez de reduzilos em número. Os Perissodactyla, em geral, perderam os dígitos um e cinco e reduziram os dígitos dois e quatro, tornando-se animais com três dedos (pata traseira da anta), ou com um único dedo, como um cavalo. Acredita-se que a redução no número de dedos relacione-se com o fato de diminuir o peso do membro para haver uma melhor aceleração e desaceleração durante o passo.

\section{Considerações finais}

A irradiação dos tapirídeos na Terra ocorreu a partir do início do Pleistoceno. Já na América do Sul, sua origem remonta ao final da época do Mioceno. Os ossos do T. terrestris são protuberantes e resistentes, com acidentes que, aparentemente, auxiliam no deslocamento cursorial. O deslocamento cursorial do T. terrestris tem grande relação com a dispersão da megaflora, estabelecendo, assim, uma interação mutualística. 


\title{
Adaptive and morphological considerations in bones of the thoracic member in Tapirus terrestris (Perissodactyla, tapiridae)
}

\begin{abstract}
Tapirus terrestris is one of the largest mammals of the Americas, it is distributed from south of Brazil and Argentina, to the north of the South American continent. This study aimed to trace a brief history of the Tapirus animals emergence using a literature review and presents environmental and morphological adaptations of the cingular scapular bones of the arm, forearm and hand of T. terrestris. We used four Tapirs skeletons donated to the Laboratory of Education and Research on Wild Animals of the Federal University of Uberlândia. The bones were carefully analyzed and described. The Tapirus animals emerged on Earth in the beginning of Pleistocene and in South America in the Miocene. The skeleton of the scapular cingulate and arm of T. terrestris consists of the scapula and humerus bones, respectively. These bones in the species studied are quite protruding and developed. The bones that constitute the skeleton of the forearm of $\mathrm{T}$. terrestris are the ulna and the radius, the bones of the hand the bones of the carpus, metacarpus, phalanges and sesamoids. The bones of $T$. terrestris are protuberant and resistant with accidents that, apparently help to cursorial displacement. As this is a cursorial animal, it has specific morphological adaptations that relates to its niche and habitat.

Keywords: Cursorial, Mammals, Descriptive morphology, Tapirus
\end{abstract} terrestris, Evolution.

\section{Referências}

CAMPBELL, B. Comparative Myology of the Forelimb of the Hippopotamus, Pig and Tapir. Teid American Jownal or Anatomy, vol. 59, N. 2, 1936.

EISENBERG, J. F. REDEFORD, K. H. 1999. Mammals of the Neotropical - The Central Neotropies: Ecuador, Peru, Bolívia, Brazil. The University of Chicago Press. Chicago. V. 3, p. 327-330

FINNEGAN, M.; MUNSON, L.; BARRER, S.; CALLE, P.P. 1993. Vesicular skin disease of tapirs. In: Proceedings of the American Association of Zoo Veterinarians. Saint Louis. pp 416-417.

FRAGOSO, J. M. V.; HUFFMAN, J. 2000. Seed-dispersal and seedling recruitment patterns by the last Neotropical megafaunal element in Amazonia, the tapir. Journal of Tropical Ecology 16: 369-385. 
GAMBARYAN, P. P. 1974. How Mammals Run: Anatomical Adaptations. New York: John Wiley and Sons.

HERNANDEZ-DIVERS, S.; QUSE, V.; MAY JR, J. A.; DE THOISY, B.; VANSTREELS, R. E. T.; MARQUES, P. A. B.; TORRES, I. L. Manual de medicina veterinária de antas em campo. IUCN/SSC TAPIR SPECIALIST GROUP (TSG). 2007.

HICKMAN, C., ROBERTS, L., LARSON, A. Princípios integrados de Zoologia. $11^{\mathrm{a}}$ ed. Rio de Janeiro: Guanabara Koogan, 2009.

HILDEBRAND, G; GOSLOW, G. Análise da Estrutura dos Vertebrados. $2^{\text {a }}$ Ed.). Editora Atheneu SP, 2006. 500 p..

HOLANDA, E. C. Os Tapiridae (Mammalia, Perissodactyla) do pleistoceno superior do estado de Rondônia, Brasil. Dissertação (Mestrado). Universidade Federal do Rio Grande do Sul. Instituto de Geociências. Programa de Pós-Graduação em Geociências. Porto Alegre, RS. 2007.

HUBBE, A. Contextualização Taxonômica e Morfométrica dos Remanescentes Ósseos da Megafauna da Gruta Cuveri (MG), Um Sitio Paleontológico do Pleistoceno Tardio. Dissertação de Mestrado - Instituto de biociências da USP. Departamento de genética e biologia evolutiva 2008. Disponível em: <http://www.teses.usp.br/teses/disponiveis/41/.../DissertacaoRevisada.pd>. Acesso em 13 de agosto de 2017.

INTERNATIONAL COMMITTEE ON VETERINARY GROSS ANATOMICAL NOMENCLATURE. Nomina anatomica veterinária. 5. ed. (rev.) Knoxville: World Association on Veterinary Anatomist, 2012, 177 p. 
MEDICI, E. P. 2001. Order Perissodactyla, Family Tapiridae (Tapirs Biology). In: Biology, medicine, and surgery of South American wild animals. Eds. FOWLER, .E. \& CUBAS, Z. S. 2001. Iowa State Univerty Press/Ames. 536p.

MEDICI, E. P. Family Tapiridae (Tapirs). In: Wilson, D. E. \& Mittermeier, R. A. Handbook of the mammals of the world - Volume 2: Hoofed Mammals. Lynx Edicions. 2011, 886p.

MOLEDO, L.; MAGNANI, E. A estrutura da Terra e a teoria da deriva continental. ComCiência . 2010, n.120 [cited 2013-10-12], s/p.

MINAYO, M. C. de S. Pesquisa Social: teoria, método e criatividade. Petrópolis: Vozes, 1994

NARANJO, E. J. P; CRUZ, E. A. Ecologia del tapir (Tapirus bairdii) en la reserva de la biosfera La Seputura, Chiapas, México. Acta Zoologica Mexicana 73:111125,1998 .

PADILLA, M.; DOWLER, R. C. Tapirus terrestris. Mammalian Species, New York, v.2, no. 481, p. 1-8, 3 figs., 1994.

POUGH, F. H.; HEISER, J. B.; MCFARLAND, W. N., 1993. A Vida dos Vertebrados. Atheneu Editora. São Paulo (SP), 839 p.

POUGH, F., JANIS, C., HEISER, J. A vida dos vertebrados. $4^{\mathrm{a}}$ ed. São Paulo: Atheneu Editora, 2008.

RADINSKY, L. B. Origin na early evolution of North Americam tapiroidea. Peabody Museum of natura history. Cannecticul. V.17, p. 1-103, 1963.

REDFORD, K. H.; J. F. EISENBERG. Mammals of the Neotropics, Volume 1.University of Chicago Press, Chicago. 1989. p 99. 
RIDLEY, M. 2006. Evolução, $3^{\text {a }}$ edição, Editora Artmed, Porto Alegre-RS.

SALGADO-LABOURIAU, M. L. 1994. História ecológica da Terra. SP, Ed. Edgard Blücher Ltda, 307 p.

STORER T. I.; USINGER R. L.; STEBBINS R. C.; NYBAKKEN J. W. 2000. Zoologia Geral. $6^{\text {a }}$ ed. Companhia Editora Nacional, São Paulo, p.642-654.

VANZOLINI, P. E. Paleoclimas e especiação em animais da América do Sul tropical. São Paulo, Estudos Avançados, 6 (15), p. 41-65, 1992.

ZORZI, B. T. Frugivoria por Tapirus terrestris em três regiões do Pantanal, Brasil (MSc thesis) (2009). Universidade Federal de Mato Grosso do Sul, Campo Grande, Brasil. 


\title{
ANEXO A - PARECER COMITÊ DE ÉTICA
}

\author{
(U) \\ Universidade Federal de Uberlândia \\ Pró-Reitoria de Pesquisa e Pós-Graduação \\ Comissão de Ética na Utilização de Animais (CEUA) \\ Avenida João Naves de Ávila, ํ‥ 2160 - Bloco A - Campus Santa Mônica - \\ Uberlândia-MG - \\ CEP 38400-089 - FONE/FAX (34) 3239-4131; e-mail:ceuaufu@yahoo.com.br; \\ www.comissoes.propp.ufu.br
}

\section{ANÁLISE FINAL № 107/12 DA COMISSÃO DE ÉTICA NA UTILIZAÇÃO DE ANIMAIS PARA O PROTOCOLO REGISTRO CEUA/UFU 069/12}

Projeto Pesquisa: "Anatomia, histologia e considerações funcionais do aparelho locomotor de tapirus terrestris linnaeus, 1758 (mammalia, perissodactyla)."

Pesquisador Responsável: Prof. Dr. André Luiz Quagliatto Santos

O protocolo não apresenta problemas de ética nas condutas de pesquisa com animais nos limites da redação e da metodologia apresentadas.

SITUAÇÃO: PROTOCOLO DE PESQUISA APROVADO.

OBS: O CEUA/UFU LEMBRA QUE QUALQUER MUDANÇA NO PROTOCOLO DEVE SER INFORMADA IMEDIATAMENTE AO CEUAA PARA FINS DE ANÁLISE E APROVAÇÃO DA MESMA.

AO FINAL DA PESQUISA DEVE SER ENTREGUE À CEUA UM RELATÓRIO. O MODELO DESTE ESTÁ NO SITE.

Uberlândia, 11 de Outubro de 2012

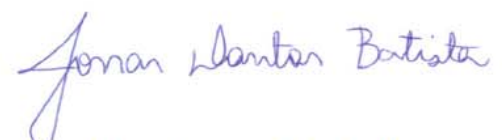

Prof. Dr. Jonas Dantas Batista Coordenador Pro tempore da CEUA/UFU 


\title{
ANEXO B - INSTRUÇÃO NORMATIVA IBAMA
}

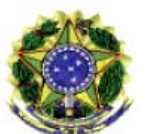 \\ SERVIÇO PÚBLICO FEDERAL \\ MINISTERIO DO MIEIO AMBIENTE \\ INSTITUTO BRASILEIRO DO MIEIO AMBIENTE E DOS RECURSOS NATURAIS RENOVÁVEIS \\ INSTRUÇÃO NORMATIVA N 154, DE 01 DE MARÇO DE 2007.
}

O PRESIDENTE ${ }^{* *}$ DO INSTITUTO BRASILEIRO DO MEIO AMBIENTE E DOS RECURSOS

NATURAIS RENOVÁVEIS - IBAMA, no uso das atribuições previstas no art. 26, inciso VI, do Anexo I, da Estrutura Regimental aprovada pelo Decreto $n^{\circ}$ 5.718, de 13 de março de 2006, e no art. 95, item VI, do Regimento Interno aprovado pela Portaria GM/MMA n 230, de 14 de maio de 2002;

Considerando as disposições das Leis nº 5.197, de 3 de janeiro de 1967, 6.938, de 31 de agosto de 1981, 9.605, de 12 de fevereiro de 1998, 9.985, de 18 de julho de 2000; Decreto-lei ${ }^{\circ} 221$, de 28 de fevereiro de 1967, Decretos n ${ }^{\circ}$ s 96.000, de 2 de maio de 1988; 99.556, de 1 de outubro de 1990; 3.179, de 21 de setembro de $1999,3.607$, de 21 de setembro de 2000 , e 4.340 , de 22 de agosto de 2002 , e;

Considerando as proposições apresentadas ao Processo Ibama n 02001.000520/2003,

\section{RE S O L E:}

Art. 26. O recolhimento e o transporte de animais encontrados mortos, para aproveitamento científico ou didático, poderão ser feitos por qualquer cidadão na ausência de autorização desde que os animais sejam destinados a instituição cientifica.

$\S 1^{\circ} \mathrm{O}$ cidadão deverá obter, sempre que possível, boletim de ocorrência junto à autoridade policial para efeito de eventual fiscalização.

$\S 2^{\circ} \mathrm{A}$ instituição cientifica deverá manter registro da entrega do animal e informar o recebimento ao Ibama por meio do Sisbio. 